\title{
Mandibular tori are associated with mandibular bone quality: a case-control study
}

\author{
N. Koç, L.B. Çağırankaya \\ Department of Dentomaxillofacial Radiology, Faculty of Dentistry, Hacettepe University, Ankara, Turkey
}

[Received: 4 September 2018; Accepted: 27 September 2018]

\begin{abstract}
Background: Torus mandibularis (TM) is one of the most common oral exostoses. The presence of TMs has been correlated with high skeletal bone mineral density. This study aimed to evaluate the relationship between TM and mandibular bone quality based on the measurement of mandibular cortical index (MCl).

Materials and methods: A case-control study was designed for patients who attended the Department of Dentomaxillofacial Radiology at the University of Hacettepe for routine dental examination. Patients with TMs were defined as cases, and those without TMs were defined as controls. The presence of tori was assessed by visual inspection and digital palpation. $\mathrm{MCl}$ assessments were done based on Klemetti's classification. The associations between the presence of TMs, $\mathrm{MCl}$, and the parafunctional activity were assessed.

Results: The sample consisted of 80 subjects with TMs and 80 control subjects. The presence of TMs was strongly associated with the parafunctional activity $(p=0.036)$ and a non-eroded mandibular cortex ( $\mathrm{MCl} C 1, p=0.001)$.

Conclusions: Parafunctional activity may be a factor related to the formation or existence of TMs. The association between TMs and mandibular morphology may suggest that subjects with TMs may have a higher mandibular bone quality compared to those without TMs. (Folia Morphol 2018; 77, 4: 736-741)
\end{abstract}

Key words: torus mandibularis, exostosis, panoramic radiography, mandibular cortical index

\section{INTRODUCTION}

Exostoses are benign growths of the bone that are considered as developmental anomalies or anatomical variations. Torus mandibularis (TM) is one of the most common oral exostoses. Mandibular tori are usually bilateral and appear at the canine-premolar region, on the lingual aspect of the mandible, above the mylohyoid ridge (Fig. 1). It is generally accepted that tori begin to develop within the third decade of life and tend to grow slowly in time [15].

The aetiology of TM is still unknown, but the postulated causes include genetic factors $[6,23]$, local environmental factors $[2,4,5,7,16,21,27]$, and systemic conditions [11, 14, 21]. It has also been hypothesized that a possible interaction between these factors may surpass a certain threshold that determines expression of the tori [10].

Osteoporosis is a systemic skeletal disease characterised by low bone mass and micro-architectural deterioration of bone tissue, leading to enhanced bone fragility and a consequent increase in fracture risk [3]. Bone mineral density (BMD) is the chief parameter to diagnose and follow-up patients with osteoporosis and is used to assess bone quantity (amount of mineral in bone tissue) [8]. BMD may also be considered as a determinant of bone quality which is particularly

Address for correspondence: Dr. N. Koç, Department of Dentomaxillofacial Radiology, Faculty of Dentistry, Hacettepe University, Sihhiye, 06230 Ankara, Turkey, tel: 90-312-3052205, fax: 90-312-3054440, e-mail: nagihan.koc@hacettepe.edu.tr 


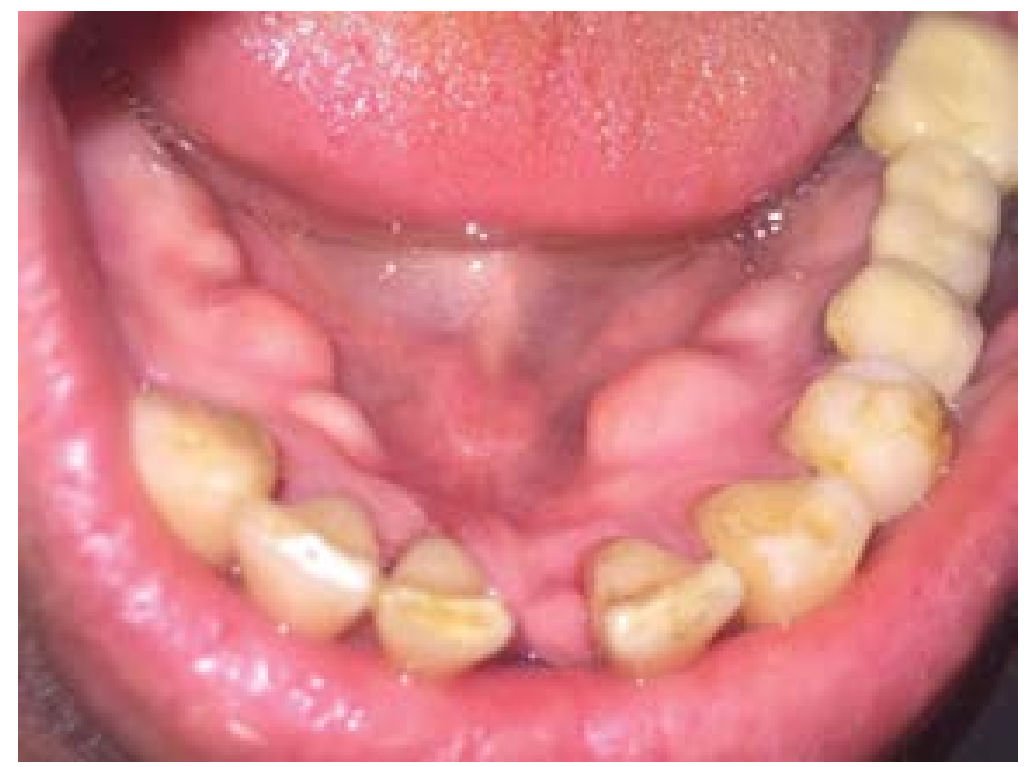

Figure 1. Bilateral mandibular tori.

important in jaw bones because of its relevance to implant planning and follow-up $[12,17]$.

Morphological changes involving the cortical layers of the mandible are shown to be dependent on age and mineral loss within the skeleton $[19,26]$. Thus, investigation of the mandible for the signs of osteoporosis is often done by evaluating the thickness and integrity of the inferior mandibular cortex. Many oral indices measured on panoramic radiographs, such as mandibular cortical index (MCl), mental index, and panoramic mandibular index have been used in dentistry to assess the mandibular bone quality and to observe signs of osteoporosis [25]. MCl is the simplest method to determine bone quality based on the classification of the radiographic appearance of the inferior mandibular cortex [18].

Numerous studies have shown associations between TM, age, number of teeth and occlusal stress as indicated by parafunctional activity $[5,7,16]$. Studies on the aetiology of TM have also focused on the effects of genetic and systemic factors to establish associations between TM, high skeletal bone density and increased bone metabolism [11, 14]. Furthermore, a significant relationship between $\mathrm{MCl}$ and skeletal bone density has been demonstrated $[13,17]$. The aim of this study was to establish whether a relationship exists between TM and mandibular bone quality expressed as $\mathrm{MCl}$-values. The authors hypothesized that the presence of TM would be associated with a non-eroded mandibular cortex. To test this hypothesis, a case-control study was conducted.

\section{MATERIALS AND METHODS}

Study design and sample

The study protocol was approved by the Non-Interventional Clinical Research Ethics Board of Hacettepe University. The study population was composed of subjects over the age of 20 years, who attended the Department of Dentomaxillofacial Radiology at the University of Hacettepe between January and June 2015 for routine dental examination. The purpose of this study and its procedures were described to the participants, and informed consent was obtained from each patient before distributing the clinical examination form. The patients presenting with TMs were defined as cases, and those without TMs were defined as controls.

\section{Inclusion and exclusion criteria}

All patients included in the study had been indicated for panoramic radiographic examination for the purpose of oral diagnosis or treatment planning. During the oral examinations, dental status of the patients was assessed based on the modified version of the Eichner index (EI) [27]. Subjects who had occlusal contacts in the premolar and molar regions of both jaws (EI Class A) were included in this study. Patients who had systemic disorders or local pathology that could affect the assessment of $\mathrm{MCl}$ or TM were excluded. Furthermore, patients who had medications that affect bone metabolism were excluded from the study. 


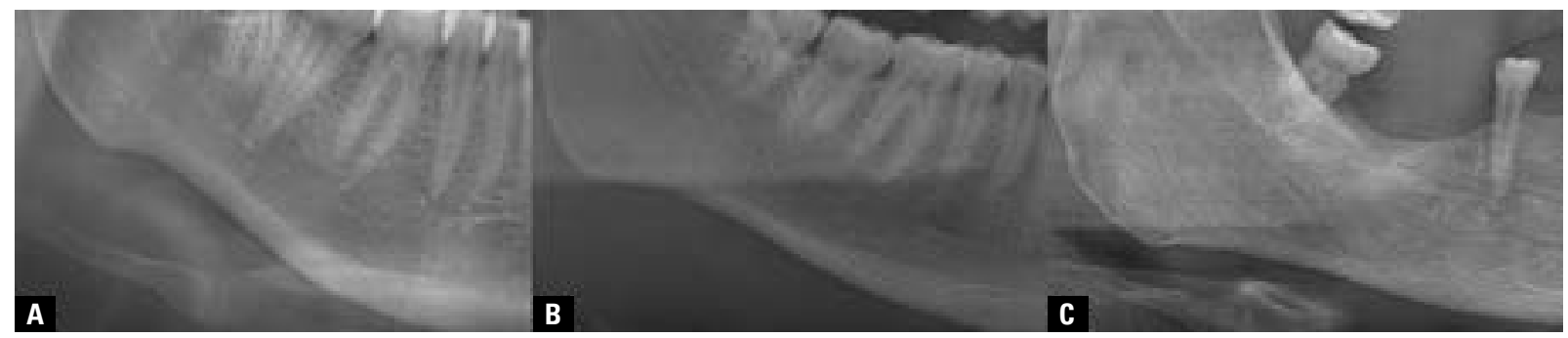

Figure 2. Mandibular cortical index; C1 (A), C2 (B) and C3 (C) categories.

\section{Definition and selection of cases and controls}

The authors identified subjects who had TMs based on the clinical examination. Each patient was clinically examined by two examiners (N.K. and L.B.C.). Both examiners were familiar with the presence of tori. The existence of TM was recorded upon verification by visual inspection and digital palpation. Small thickenings or a questionable TM were considered as a normal lingual outline.

Control group consisted of subjects without TM who were randomly selected from the same population. Control subjects were similar to cases in terms of age and gender. Subjects were excluded from the control group if they had TM.

The primary outcome variable of the present study was the presence of TMs. The following predictor variables were recorded: age, gender, $\mathrm{MCl}$, and the parafunctional activity.

\section{Data collection}

Parafunctional activity. Parafunctional habits investigated in this study were diurnal and nocturnal bruxism. The patients were interviewed to determine if they had an awareness of clenching or grinding during the daytime or were informed about clenching or grinding sound during sleep by their partners or others $[16,27]$. The report of the presence of any of these criteria were considered positive for the parafunctional activity.

Radiographic analysis. Panoramic images of the patients were obtained by an Orthophos XG 5 (Sirona Dental Systems, Bensheim, Germany) and a Veraview IC5 HD (J. Morita MFG. Corp., Kyoto, Japan) panoramic machine. Images were transferred to the digital archiving system (Turcasoft Software Ltd., Samsun, Turkey).

Radiographs with poor density and contrast and with patient positioning or head alignment errors were excluded from the study. Radiographic analysis was performed independently by two oral radiol- ogists (L.B.Ç. and N.K.) after completing all clinical examinations.

The morphology of the mandibular inferior cortex was determined by observing both sides of the mandible distally from the mental foramen according to the criteria defined by Klemetti et al. [18] (Fig. 2):

- C1: The endosteal margin of the cortex is even and sharp on both sides;

- C2: The endosteal margin of the cortex shows semilunar defects (resorption cavities) with cortical residues one to three layers thick on one or both sides;

- C3: The cortical layer contains heavy endosteal cortical residues and is clearly porous.

For the evaluation of the intra- and inter-observer variability, 40 radiographs were randomly selected, and $\mathrm{MCl}$ measurements were repeated at the interval of 2 weeks. The Kappa statistic was used to evaluate intra- and inter-observer agreements.

\section{Statistical analysis}

The statistical analyses were performed using IBM SPSS Statistics for Windows, version 17.0 (SPSS Inc., Chicago, IL, USA). The independent samples t-test was used to compare the age distribution of two groups. Associations between TM and each of the variables were assessed based on the $\chi^{2}$ test. Variables with a significance level of less than 0.20 were selected for unconditional logistic regression analysis [5]. The Hosmer-Lemeshow test was used to analyse the model adjustment. Risk estimates were calculated and presented as odds ratios (ORs) with $95 \%$ confidence intervals (Cls). Results were considered significant if $p<0.05$.

\section{RESULTS}

The study sample consisted of 80 subjects with TMs (30 male and 50 female) and 80 control subjects (36 male and 44 female). The case group had a mean age of $35.7 \pm 11.5$ years (range: $21-68$ years) where- 
as the control group had $36.1 \pm 11.3$ years (range: 21-68 years). No significant difference was found between groups by age ( $\chi^{2}$ test, $p=0.88$ ) or gender $\left(\chi^{2}\right.$ test, $\left.p=0.42\right)$. The characteristics of the case and control groups are presented in Table 1.

There were two categories of $\mathrm{MCl}$ detected: C1 $(n=35 ; 21.9 \%)$ and C2 $(n=125 ; 78.1 \%)$. Regarding $\mathrm{MCl}$ assessments, the Kappa values for the intra-observer agreements were 0.93 and 0.94 . The Kappa values for the inter-observer agreements in the first and second sessions were 0.82 and 0.93 , respectively.

Forty-seven (29.4\%) subjects were classified as having parafunctional activity. Significant differences were observed between case and control groups regarding parafunctional activity ( $p=0.005$; Table 1$)$ and $\mathrm{MCl}(\mathrm{p}<0.001)$.

Logistic regression analysis revealed that the presence of TMs was strongly associated with the parafunctional activity (Table 2; OR 2.25; 95\% Cl 1.03-4.90; $\mathrm{p}=0.036)$ and a non-eroded mandibular cortex ( $\mathrm{MCl}$ C1; OR 6.26; 95\% Cl 2.30-17.05, p < 0.001). Although the variables "age" and "gender" showed no significance, they were kept in the model as the adjustment worsened when they were removed (Hosmer-Lemeshow test; $\chi^{2}$ value $=3.67, p=0.72$ ).

\section{DISCUSSION}

The main objective of the present study was to identify whether there is an association between the presence of TM and the mandibular bone quality which was expressed as $\mathrm{MCl}$. Results of this study confirmed our hypothesis that the presence of TMs is associated with non-eroded mandibular cortices (C1 category of $\mathrm{MCl}$ ). This finding is important because there has been very little research reported on the association between TM and mandibular morphology $[1,4,24]$. The present study also provided information about the relationship between TM and the parafunctional activity, which has been demonstrated by numerous studies $[4,5,16,21]$.

In previous studies on the prevalence of TM, age and the number of teeth were found to be related to the presence of TMs $[7,22]$. In the present study, the cases (subjects with TMs) and controls (subjects without TMs) were similar in age, gender, and dental status, which provided controlling for the potential effects of these variables. Nevertheless, regarding the relationship between gender and TM, results from a study in a Turkish population showing similar detection rates of TM between males and females
Table 1. Characteristics of case subjects and controls

\begin{tabular}{lccc}
\hline Characteristic & $\begin{array}{c}\text { Cases } \\
\text { (n= 80) }\end{array}$ & $\begin{array}{c}\text { Controls } \\
(\mathbf{n}=\mathbf{8 0})\end{array}$ & $\mathbf{P}$ \\
\hline Age [years]: & & & 0.887 \\
$21-30$ & 30 & 28 & \\
$31-40$ & 28 & 27 & \\
$41-50$ & 11 & 17 & \\
$>50$ & 11 & 8 & \\
Gender: & & & 0.423 \\
$\quad$ Female & 50 & 44 & \\
$\quad$ Male & 30 & 36 & \\
Parafunctional activity: & & & 0.005 \\
$\quad$ Yes & 32 & 15 & \\
No & 48 & 65 & \\
Mandibular cortical index: & & & $<0.001$ \\
$\quad$ Non-eroded (C1) & 29 & 6 & \\
Eroded (C2) & 51 & 74 & \\
\hline
\end{tabular}

Table 2. Odds ratios for the association of variables with presence of torus mandibularis

\begin{tabular}{lccc}
\hline Variables & $\mathbf{P}$ & $\begin{array}{c}\text { Odds } \\
\text { ratio }\end{array}$ & $\begin{array}{c}\text { 95\% confidence } \\
\text { interval }\end{array}$ \\
\hline $\begin{array}{l}\text { Age [years]: } \\
\quad \leq 40 \\
>40\end{array}$ & 0.872 & 1.06 & $0.50-2.22$ \\
Gender: & & 1 & \\
$\quad$ Female & & & \\
$\quad$ Male & 0.602 & 0.83 & $0.41-1.67$ \\
$\begin{array}{l}\text { Parafunctional activity: } \\
\quad \text { Yes }\end{array}$ & & 1 & \\
$\quad$ No & 0.036 & 2.25 & $1.03-4.90$ \\
Mandibular cortical index: & & 1 & \\
$\quad$ & & & \\
Non-eroded (C1) & $<0.001$ & 6.26 & $2.30-17.05$ \\
$\quad$ Eroded (C2) & & 1 & \\
\hline
\end{tabular}

suggest that sex has an insignificant effect on the prevalence of TM [24].

The reason behind the selection of the patients over the age of 20 years in this study was related to the hypothesis that tori begin to develop within the third decade of the life, that is, approximately the time that peak BMD is reached.

Mandibular cortical index is a simple method to assess mandibular morphology and the possible signs of osteoporosis based on the evaluation of the cortical shape of the mandible [18]. The use of the $\mathrm{MCl}$ scale (C1-3) in determining a low bone mass and in the 
assessment of the quality of the mandibular bone has been validated as a surrogate for BMD measurements: a non-eroded cortex ( $\mathrm{C} 1$ category of $\mathrm{MCl}$ ) is indicative of high systemic and mandibular bone density [13, 18]. The present study demonstrated excellent observer agreements regarding the $\mathrm{MCl}$ assessments. This finding is consistent with previous studies reporting $\mathrm{MCl}$ is highly repeatable $[9,18,20]$.

The $\mathrm{C} 3$ category of $\mathrm{MCl}$ was not observed in this study, which might be related to the age characteristics of the sample (mean age: $35.9 \pm 11.3$ years). Because the first signs of bone loss start around the age of 30 years, age-related changes in bone might not be reflected in this patient sample yet.

Consistent with the literature, the present study has also found an association between parafunctional activity and the presence of TMs $[4,5,16]$. This finding supports the suggestion that parafunctional stresses may be involved in the formation or existence of TMs $[4,5]$.

The interaction demonstrated between TM and $\mathrm{MCl}$ in this study may imply that developmental or environmental factors involved in the regulation of bone quality could play a role in the formation or existence of TM. To our knowledge, only two studies have examined the relationship between TM and MCl $[4,24]$. Results of this study showing an association between the presence of TM and a non-eroded mandibular cortex ( $\mathrm{C} 1$ category of $\mathrm{MCl}$ ) is compatible with the findings of a case-control study by Cortes et al. [4]. However, the authors did not describe a method that had been used for controlling the effect of dentition in their study. The study of Uysal et al. [24] used a cross-sectional design to investigate the relationship between $\mathrm{TM}$ and $\mathrm{MCl}$, but failed to establish an association. A case-control study [1] was intended to investigate the association between $\mathrm{TM}$ and $\mathrm{MCl}$ but used a different method to evaluate $\mathrm{MCl}$, which refers to the panoramic mandibular index [20].

Although recent knowledge about TM implicates the role of genetic and local factors on the formation and the existence of tori, the exact aetiology of these overgrowths remain unclear. Our findings showing an association between the presence of TM and a non-eroded mandibular cortex (C1 category of $\mathrm{MCl}$ ) suggest that subjects with TM may have a higher mandibular bone quality compared to subjects without TM. The assessment of the presence of TMs as a potential indicator of mandibular bone quality might be useful in treatment planning for implants and regenerative procedures.

\section{Limitations of this study}

Limitations of this study include its case-control design which can only provide evidence of an association but not a causal relationship. Further longitudinal studies are needed to explore whether a subject with parafunctional activity would develop TM or not. Furthermore, a direct evaluation of bone quality (such as biochemical markers, microradiography, or histomorphometry) was not performed in this study.

\section{CONCLUSIONS}

Parafunctional activity may be a factor related to the formation or existence of TM. The association between TM and mandibular morphology may suggest that subjects with mandibular tori may have a higher mandibular bone quality compared to those without TMs.

\section{REFERENCES}

1. Al-Dwairi ZN, Al-Daqaq ANF, Kielbassa AM, et al. Association between oral tori, occlusal force, and mandibular cortical index. Quintessence Int. 2017; 48(10): 841-849, doi: 10.3290/j.qi.a38856, indexed in Pubmed: 28849805.

2. Çağırankaya B, Hatipoğlu M, Kansu Ö. Is there an association between torus mandibularis and bite force? J Hacettepe Univ Fac Dent. 2005; 29(4): 15-17.

3. Consensus development conference: Diagnosis, prophylaxis, and treatment of osteoporosis. Am J Med. 1993; 94(6): 646-650, doi: 10.1016/0002-9343(93)90218-e.

4. Cortes AR, Jin Z, Morrison MD, et al. Mandibular tori are associated with mechanical stress and mandibular shape. J Oral Maxillofac Surg. 2014; 72(11): 2115-2125, doi: 10.1016/j.joms.2014.05.024, indexed in Pubmed: 25239215.

5. Canto GD, Freitas ST, Filho GS, et al. Association between mandibular torus and parafunctional activity. Int J Stomat Occ Med. 2012; 6(2): 43-49, doi:10.1007/s12548-0120064-5.

6. Eggen S. Torus mandibularis: an estimation of the degree of genetic determination. Acta Odontol Scand. 1989; 47(6): 409-415, doi:10.3109/00016358909004810, indexed in Pubmed: 2609949.

7. Eggen S, Natvig B. Relationship between torus mandibularis and number of present teeth. Scand J Dent Res. 1986; 94(3): 233-240, doi:10.1111/j.1600-0722.1986. tb01758.x, indexed in Pubmed: 3461543.

8. Fonseca $\mathrm{H}$, Moreira-Gonçalves $\mathrm{D}$, Coriolano HJA, et al. Bone quality: the determinants of bone strength and fragility. Sports Med. 2014; 44(1): 37-53, doi: 10.1007/ s40279-013-0100-7, indexed in Pubmed: 24092631.

9. Gulsahi A, Yüzügüllü B, Imirzalioglu P, et al. Assessment of panoramic radiomorphometric indices in Turkish patients of different age groups, gender and dental status. Dentomaxillofac Radiol. 2008; 37(5): 288-292, doi: 10.1259/ dmfr/19491030, indexed in Pubmed: 18606751.

10. Haugen LK. Palatine and mandibular tori. Amorphologic study in the current Norwegian population. Acta Odontol Scand. 1992; 50(2): 65-77, doi:10.3109/00016359209012748, indexed in Pubmed: 1604967. 
11. Hjertstedt J, Burns EA, Fleming R, et al. Mandibular and palatal tori, bone mineral density, and salivary cortisol in community-dwelling elderly men and women. J Gerontol A Biol Sci Med Sci. 2001; 56(11): M731-M735, doi: 10.1093/ gerona/56.11.M731, indexed in Pubmed: 11682583.

12. Horner K, Devlin H. The relationship between mandibular bone mineral density and panoramic radiographic measurements. J Dent. 1998; 26(4): 337-343, doi: 10.1016/ S0300-5712(97)00020-1, indexed in Pubmed: 9611939.

13. Horner K, Devlin H. The relationships between two indices of mandibular bone quality and bone mineral density measured by dual energy X-ray absorptiometry. Dentomaxillofac Radiol. 1998; 27(1): 17-21, doi: 10.1038/ sj.dmfr.4600307, indexed in Pubmed: 9482017.

14. Hosoi T, Yoda $\mathrm{T}$, Yamaguchi $\mathrm{M}$, et al. Elderly women with oral exostoses had higher bone mineral density. J Bone Miner Metab. 2003; 21(2): 120-122, doi: 10.1007/ s007740300020, indexed in Pubmed: 12601578.

15. Hrdlička A. Mandibular and maxillary hyperostoses. Am J Phys Anthropol. 1940; 27(1): 1-67, doi: 10.1002/ ajpa.1330270127.

16. Kerdpon D, Sirirungrojying S. A clinical study of oral tori in southern Thailand: prevalence and the relation to parafunctional activity. Eur J Oral Sci. 1999; 107(1): 9-13, doi: 10.1046/j.0909-8836.1999.eos107103.x, indexed in Pubmed: 10102745.

17. Klemetti E, Kolmakow S. Morphology of the mandibular cortex on panoramic radiographs as an indicator of bone quality. Dentomaxillofac Radiol. 1997; 26(1): 22-25, doi: 10.1038/sj.dmfr.4600203, indexed in Pubmed: 9446986

18. Klemetti E, Kolmakov S, Kröger H. Pantomography in assessment of the osteoporosis risk group. Scand J Dent Res. 1994; 102(1): 68-72, doi:0.1111/j.1600-0722.1994. tb01156.x, indexed in Pubmed: 8153584.

19. Klemetti E, Vainio P, Lassila V, et al. Cortical bone mineral density in the mandible and osteoporosis status in post- menopausal women. Scand J Dent Res. 1993; 101(4): 219-223, doi: 10.1111/j.1600-0722.1993.tb01108.x, indexed in Pubmed: 8362200.

20. Ledgerton D, Horner K, Devlin H, et al. Radiomorphometric indices of the mandible in a British female population. Dentomaxillofac Radiol. 1999; 28(3): 173-181, doi: 10.1038/ sj/dmfr/4600435, indexed in Pubmed: 10740473.

21. Morrison MD, Tamimi F. Oral tori are associated with local mechanical and systemic factors: a case-control study. J Oral Maxillofac Surg. 2013; 71(1): 14-22, doi: 10.1016/j. joms.2012.08.005, indexed in Pubmed: 23010373.

22. Sonnier KE, Horning GM, Cohen ME. Palatal tubercles, palatal tori, and mandibular tori: prevalence and anatomical features in a U.S. population. J Periodontol. 1999; 70(3): 329-336, doi: 10.1902/jop.1999.70.3.329, indexed in Pubmed: 10225550.

23. Suzuki M, Sakai T. A familial study of torus palatinus and torus mandibularis. Am J Phys Anthropol. 1960; 18(4): 263-272, doi:10.1002/ajpa.1330180404.

24. Uysal S, Cağirankaya BL, Hatipoğlu MG. Do gender and torus mandibularis affect mandibular cortical index? A cross-sectional study. Head Face Med. 2007; 3: 37, doi: 10.1186/1746160X-3-37, indexed in Pubmed: 17971202.

25. Vlasiadis KZ, Skouteris CA, Velegrakis GA, et al. Mandibular radiomorphometric measurements as indicators of possible osteoporosis in postmenopausal women. Maturitas. 2007; 58(3): 226-235, doi: 10.1016/j.maturitas.2007.08.014, indexed in Pubmed: 17923346.

26. von Wowern N, Stoltze K. Sex and age differences in bone morphology of mandibles. Scand J Dent Res. 1978; 86(6): 478-485, doi: 10.1111/j.1600-0722.1978.tb00654.x, indexed in Pubmed: 284570.

27. Yoshinaka M, Ikebe K, Furuya-Yoshinaka M, et al. Prevalence of torus mandibularis among a group of elderly Japanese and its relationship with occlusal force. Gerodontology. 2014; 31(2): 117-122, doi: 10.1111/ger.12017, indexed in Pubmed: 23167776. 